\title{
Usefulness of Age-Stratified N-Terminal Prohormone of Brain Natriuretic Peptide for Diagnosing Kawasaki Disease
}

\author{
Sang Hoon Lee, ${ }^{1}$ Eun Song Song, ${ }^{1}$ Somy Yoon, ${ }^{2}$ Seunghee Hong, ${ }^{1}$ Hwa Jin Cho, ${ }^{1}$ \\ Eun Mi Yang, ${ }^{1}$ Gwang Hyeon Eom, ${ }^{2}$ Gaeun Kang, ${ }^{3}$ and Young Kuk Cho ${ }^{1}$ \\ ${ }^{1}$ Department of Pediatrics, Chonnam National University Medical School, Chonnam National University Hospital, Gwangju 61469, \\ Republic of Korea \\ ${ }^{2}$ Department of Pharmacology, Chonnam National University Medical School, Gwangju 61469, Republic of Korea \\ ${ }^{3}$ Division of Clinical Pharmacology, Chonnam National University Hospital, Gwangju 61469, Republic of Korea
}

Correspondence should be addressed to Gaeun Kang; gaeun.kang@gmail.com and Young Kuk Cho; youngcx@hanmail.net

Received 14 July 2017; Accepted 25 September 2017; Published 22 November 2017

Academic Editor: Juan Bueno

Copyright ( 2017 Sang Hoon Lee et al. This is an open access article distributed under the Creative Commons Attribution License, which permits unrestricted use, distribution, and reproduction in any medium, provided the original work is properly cited.

$\mathrm{N}$-terminal prohormone of brain natriuretic peptide (NT-proBNP) was recently reported as a biomarker for diagnosing Kawasaki disease (KD). The basal NT-proBNP level, however, gradually decreases with age. We investigated the usefulness of an age-stratified cutoff value of NT-proBNP for diagnosing KD. All the patients enrolled in this study visited Chonnam National University Hospital between December 2007 and March 2016. The KD groups consisted of 214 patients with complete KD and 129 patients with incomplete KD. The control group included 62 children with simple febrile illness but without heart disease. Laboratory data including NT-proBNP level were evaluated. Each group was divided into subgroups according to patient age $(<6$ months, 6-12 months, 12-24 months, and >24 months), and different cutoff values of NT-proBNP were calculated. The cutoff values of NTproBNP used to diagnose total $\mathrm{KD}$ and incomplete $\mathrm{KD}$ were 762 and $762 \mathrm{pg} / \mathrm{mL}$ (<6 months), 310 and $310 \mathrm{pg} / \mathrm{mL}(6-12$ months), 326 and $326 \mathrm{pg} / \mathrm{mL}$ (12-24 months), and 208 and $137 \mathrm{pg} / \mathrm{mL}$ (>24 months), respectively. In conclusion, age-stratified NT-proBNP is a useful biomarker for the differential diagnosis of KD in patients with a simple febrile illness.

\section{Introduction}

Kawasaki disease $(\mathrm{KD})$ is a systemic vasculitis that usually affects children and infants $<5$ years of age $[1,2]$. KD is thought to be the most common cause of acquired heart disease [3]. Among untreated patients or those subject to a treatment delay, $20-25 \%$ suffers from complications of coronary artery disease $[3,4]$. Therefore, a prompt and accurate diagnosis and timely treatment of $\mathrm{KD}$ with intravenous immunoglobulin (IVIg) are necessary to minimize the complications associated with $\mathrm{KD}$ [3].

Because $\mathrm{KD}$ can only be diagnosed based on clinical symptoms, the diagnosis and treatment of $\mathrm{KD}$ patients are sometimes delayed; this is especially true for patients with incomplete $\mathrm{KD}$, which is characterized by an incomplete presentation of diagnostic clinical symptoms [3]. Therefore, in cases of vague clinical symptoms, the diagnosis inevitably depends on adjuvant diagnostic markers. Some that are already broadly adopted are elevated C-reactive protein (CRP), elevated erythrocyte sedimentation rate (ESR), low albumin, anemia, elevation of alanine aminotransferase (ALT), a high platelet count after 7 days, and a high white blood cell count or pyuria [5].

$\mathrm{N}$-terminal prohormone of brain natriuretic peptide (NT-proBNP) is an adjuvant marker used to diagnose KD, and many studies have illustrated its usefulness $[6,7]$. Lee et al. reported that the cutoff value of NT-proBNP for diagnosing incomplete $\mathrm{KD}$ was $158 \mathrm{pg} / \mathrm{dL}$, with $81 \%$ sensitivity and $74 \%$ specificity [6]. Another study reported that the cutoff value of NT-proBNP used to diagnose KD was $260 \mathrm{pg} / \mathrm{dL}$, which displayed $93 \%$ sensitivity and $88 \%$ specificity [7]. However, the normal range of NT-proBNP varies widely with age [8]. The levels of NT-proBNP were the highest in the first few days of life, showed a marked 
decline in the first week, and continued to decline gradually with age [8]. Because of the natural age-based differences, applying the same cutoff value of NT-proBNP to each patient regardless of age would be unreasonable. Here, we investigated NT-proBNP as a biomarker by age in patients with $\mathrm{KD}$ or simple febrile illness and checked the usefulness of age-stratified cutoff values of NT-proBNP for diagnosing KD.

\section{Methods}

2.1. Patients. The two KD groups (incomplete and complete $\mathrm{KD}$ ) included a total of 343 children who were diagnosed with KD and treated with IVIg at Chonnam National University Hospital between December 2007 and March 2016. Complete $\mathrm{KD}$ was diagnosed according to American Heart Association criteria suggested in 2017 [5]. The criteria include fever persisting for at least 5 days and fulfillment of at least four of five clinical symptoms [5]. In contrast, incomplete $\mathrm{KD}$ was diagnosed when a patient presented three or fewer symptoms and other possible causes of the fever were excluded [5]. The control group included 62 age-matched children who visited our center for an evaluation of febrile illness and underwent laboratory testing for NT-proBNP; we excluded those with heart diseases such as congenital heart disease, cardiomyopathy, and acute myocarditis, which can affect NT-proBNP levels [9]. Serum samples were obtained to measure serum NT-proBNP levels on the day that IVIg was started in patients with complete and incomplete KD and on the first day of admission in the control group. Serum NT-proBNP was analyzed using an electrochemiluminescence immunoassay (ElecsysProBNP Sandwich Immunoassay; Roche Diagnostics, Basel, Switzerland). At the same time, complete blood cell count (CBC); ESR; and levels of $\mathrm{CRP}$, total protein, albumin, blood urea nitrogen (BUN), creatinine, aspartate aminotransferase (AST), ALT, creatine kinase (CK), CK-MB, myoglobin, and troponin-I were also obtained and analyzed. The reference values of NT-proBNP suggested by age were highest at birth and then significantly decreased over the first 2 years, especially before the age of 1 year. It showed little change with age after 2 years of age [8]. Therefore, we classified patients with $\mathrm{KD}$ and control patients into four subgroups by age considering the nature of these normal changes with age of NT-proBNP ( $<6$ months, 6-12 months, $12-24$ months, and $>24$ months).

2.2. Statistics. SPSS (version 23.0; SPSS, Chicago, IL, USA) was used for the data analyses. Continuous variables are presented as means \pm standard deviations. The chi-square test was used to assess the statistical significance of differences between the independent variables. To compare data among more than three groups, analysis of variance (ANOVA) was employed, followed by Tukey's honestly significant difference post hoc test. When the homogeneity of variance was not present according to Levene's test, Dunnett's T3 was used for the post hoc test. To evaluate the correlation between two serologic factors, both Pearson's test and Spearman's rho test were utilized. The KD groups were evaluated by multinomial logistic regression including NT-proBNP, CRP, platelet count, and albumin as fixed effects. When the normal distribution was not assumed on the Shapiro-Wilk test, the Kruskal-Wallis test followed by the Bonferroni correction was used. The cutoff values for diagnosing KD were obtained using receiver operating characteristic (ROC) curve analysis. $P$ values $<0.05$ were considered statistically significant.

2.3. Ethics Statement. The purpose of this study was explained in detail to each patient's parent, who provided informed consent after careful consideration. Approval for the present study was obtained from the Institutional Review Board of Chonnam National University Hospital (protocol number I-2009-09-103). All data were treated confidentially.

\section{Results}

3.1. Patient Characteristics. Patient demographics are shown in Table 1. The total number of KD patients was 343, which included complete KD patients $(n=214,62.4 \%)$ and incomplete $\mathrm{KD}$ patients $(n=129,37.6 \%)$. In the complete $\mathrm{KD}$ group, the male-to-female ratio was 1.53:1 (136 versus 78) and the mean patient age was $2.8 \pm 1.8$ years. The complete $\mathrm{KD}$ group was classified into four subgroups by age ( $<6$ months $[n=21,9.8 \%], 6-12$ months $[n=20,9.3 \%]$, $12-24$ months $[n=39,18.2 \%]$, and $>24$ months $[n=134$, $62.6 \%$ ) (Table 2). In the incomplete KD group, the maleto-female ratio was 1.39:1 (75 versus 54) and the mean patient age was $2.3 \pm 2.0$ years. The incomplete $\mathrm{KD}$ group was divided into four subgroups ( $<6$ months $[n=25$, 19.4\%], 6-12 months [ $n=22,17.1 \%], 12-24$ months $[n=$ $21,16.3 \%]$, and $>24$ months $[n=61,47.3 \%]$ ) (Table 2). Sixty-two age-matched febrile illness patients were enrolled as controls; the male-to-female ratio was 3.13:1 (47 versus $15)$ and the mean age was $2.9 \pm 2.7$ years. Neither sex nor fever duration differed significantly among the three groups. There were no significant differences in measurement time of NT-proBNP in patients with complete KD (4.2 \pm 1.9 days), patients with incomplete $\mathrm{KD}(4.5 \pm 2.5$ days), and simple febrile illness controls ( $4.8 \pm 3.9$ days, $P=0.133)$. The clinical diagnoses in the control group were as follows: pneumonia $(n=19)$, acute tonsillitis $(n=15)$, cervical lymphadenitis $(n=9)$, acute bronchiolitis $(n=6)$, urinary tract infection $(n=5)$, acute cellulitis $(n=3)$, acute epiglottitis $(n=2)$, bacteremia $(n=2)$, and bacterial keratoconjunctivitis $(n=1)$. Nine patients yielded positive bacterial blood cultures. The control group was classified into four subgroups based on age (<6 months $[n=8,12.9 \%], 6-12$ months $[n=11$, $17.7 \%], 12-24$ months $[n=12,19.4 \%]$, and $>24$ months $[n=31,50.0 \%])$ (Table 2).

3.2. Laboratory Findings at Diagnosis in Patients with KD and Simple Febrile Illness. Laboratory data from the complete and incomplete $\mathrm{KD}$ groups and the febrile control group at the time of diagnosis are shown in Figure 1 and Tables 1 and 2. At the time of diagnosis, the white blood cell count in complete KD patients $\left(14,561 \pm 4855 / \mathrm{mm}^{3}\right)$ was significantly higher than that in febrile illness controls $(12,268 \pm$ $4753 / \mathrm{mm}^{3}, P=0.007$ ) (Figure 1(a)). The percentage of neutrophils in complete KD patients $(66.3 \pm 15.7 \%)$ was significantly higher than those in incomplete KD patients $(56.8 \pm$ 
TABLE 1: Demographic characteristics and laboratory findings at diagnosis in patients with complete Kawasaki disease (KD), incomplete KD, and simple febrile illness (control).

\begin{tabular}{|c|c|c|c|c|c|c|}
\hline & \multicolumn{2}{|c|}{ Complete KD } & \multicolumn{2}{|c|}{ Incomplete KD } & \multicolumn{2}{|r|}{ Control } \\
\hline & $n$ & Value & $n$ & Value & $n$ & Value \\
\hline Age (years) & 214 & $2.8 \pm 1.8$ & 129 & $2.3 \pm 2.0$ & 62 & $2.9 \pm 2.7$ \\
\hline Sex (boy/girl) & 214 & $136 / 78$ & 129 & $75 / 54$ & 62 & $47 / 15$ \\
\hline Total fever duration (days) & 214 & $6.0 \pm 1.8$ & 129 & $6.3 \pm 2.5$ & 62 & $5.3 \pm 4.5$ \\
\hline Duration of fever until IVIg (days) & 214 & $4.2 \pm 1.9$ & 129 & $4.5 \pm 2.5$ & 62 & $4.8 \pm 3.9$ \\
\hline White blood cell (count $/ \mathrm{mm}^{3}$ ) & 214 & $14,561 \pm 4855$ & 129 & $13,955 \pm 5557$ & 62 & $12,268 \pm 4753^{\dagger}$ \\
\hline Neutrophil (\%) & 213 & $66.3 \pm 15.7$ & 129 & $56.8 \pm 17.3^{\dagger}$ & 62 & $56.0 \pm 21.0^{\dagger}$ \\
\hline Hemoglobin (g/dL) & 214 & $11.4 \pm 1.1$ & 129 & $11.2 \pm 1.2$ & 62 & $11.5 \pm 1.3$ \\
\hline Platelet $\left(\times 10^{3} / \mathrm{mm}^{3}\right)$ & 214 & $343 \pm 104$ & 129 & $370 \pm 145$ & 62 & $279 \pm 97^{\dagger, \ddagger}$ \\
\hline Erythrocyte sedimentation rate $(\mathrm{mm} / \mathrm{h})$ & 89 & $64.3 \pm 26.6$ & 50 & $69.3 \pm 32.8$ & 21 & $34.7 \pm 32.0^{\dagger, \ddagger}$ \\
\hline C-reactive protein $(\mathrm{mg} / \mathrm{dL})$ & 214 & $8.1 \pm 5.3$ & 129 & $7.0 \pm 5.6$ & 62 & $4.8 \pm 5.0^{\dagger, 9}$ \\
\hline Aspartate aminotransferase (U/L) & 212 & $92 \pm 138$ & 128 & $66 \pm 104$ & 62 & $85 \pm 132$ \\
\hline Alanine aminotransferase (U/L) & 212 & $111.3 \pm 170.8$ & 128 & $67.8 \pm 112.9^{*}$ & 62 & $63.4 \pm 131.8$ \\
\hline Total bilirubin (mg/dL) & 139 & $1.1 \pm 1.4$ & 82 & $0.7 \pm 0.8^{*}$ & 28 & $0.6 \pm 0.4$ \\
\hline Total protein $(\mathrm{g} / \mathrm{dL})$ & 198 & $6.4 \pm 0.8$ & 117 & $6.5 \pm 0.7$ & 61 & $6.5 \pm 0.6$ \\
\hline Albumin (g/dL) & 214 & $3.6 \pm 0.6$ & 129 & $3.7 \pm 0.5$ & 62 & $3.9 \pm 0.4^{\dagger, g}$ \\
\hline Lactate dehydrogenase (U/L) & 81 & $623 \pm 160$ & 53 & $638 \pm 276$ & 24 & $745 \pm 258$ \\
\hline Creatine kinase (IU/L) & 181 & $151 \pm 376$ & 114 & $120 \pm 248$ & 60 & $272 \pm 503^{9}$ \\
\hline Creatine kinase-MB (ng/mL) & 206 & $7.3 \pm 10.5$ & 122 & $4.8 \pm 8.3$ & 57 & $12.2 \pm 11.9^{\dagger, \ddagger}$ \\
\hline Myoglobin (ng/mL) & 130 & $25.4 \pm 60.2$ & 85 & $19.0 \pm 18.6$ & 26 & $74.0 \pm 92.4^{\dagger, \ddagger}$ \\
\hline Troponin I (ng/mL) & 197 & $0.02 \pm 0.04$ & 115 & $0.01 \pm 0.01$ & 45 & $0.01 \pm 0.01$ \\
\hline N-terminal prohormone of brain natriuretic peptide $(\mathrm{pg} / \mathrm{mL})$ & 214 & $1785 \pm 2858$ & 129 & $1153 \pm 1725^{*}$ & 62 & $311 \pm 400^{\dagger, 9}$ \\
\hline
\end{tabular}

IVIg: intravenous immunoglobulin; KD: Kawasaki disease. Data are shown as mean $\pm \mathrm{SD}$. ${ }^{*} P<0.05 .{ }^{\dagger} P<0.01$ versus complete KD. ${ }^{9} P<0.05$. ${ }^{\ddagger} P<0.01$ versus incomplete KD.

$17.3 \%, P<0.001)$ and febrile illness controls $(56.0 \pm 21.0 \%$, $P=0.002$ ) (Figure 1(b)). The platelet count in both complete $\mathrm{KD}$ patients $\left(343 \pm 104 \times 10^{3} / \mathrm{mm}^{3}\right)$ and incomplete $\mathrm{KD}$ patients $\left(370 \pm 145 \times 10^{3} / \mathrm{mm}^{3}\right)$ was significantly higher than that in controls $\left(279 \pm 97 \times 10^{3} / \mathrm{mm}^{3}, P<0.001\right.$ for both) (Figure $1(\mathrm{c}))$. The ESR in complete $\mathrm{KD}$ patients $(64.3 \pm$ $26.6 \mathrm{~mm} / \mathrm{h})$ and in incomplete $\mathrm{KD}$ patients $(69.3 \pm$ $32.8 \mathrm{~mm} / \mathrm{h}$ ) was also significantly higher than that in controls $(34.7 \pm 32.0 \mathrm{~mm} / \mathrm{h}, P<0.001$ for both) (Figure $1(\mathrm{~d})$ ). The CRP level in complete KD patients $(8.1 \pm 5.3 \mathrm{mg} / \mathrm{dL})$ and incomplete $\mathrm{KD}$ patients $(7.0 \pm 5.6 \mathrm{mg} / \mathrm{dL})$ was also significantly higher than that in febrile illness controls $(4.8 \pm$ $5.0 \mathrm{mg} / \mathrm{dL}, P<0.001$ and $P=0.039$, resp.) (Figure $1(\mathrm{e})$ ). Serum albumin levels in complete KD patients $(3.6 \pm 0.6 \mathrm{~g} /$ $\mathrm{dL})$ and incomplete KD patients $(3.7 \pm 0.5 \mathrm{~g} / \mathrm{dL})$ were significantly lower than that in controls $(3.9 \pm 0.4 \mathrm{~g} / \mathrm{dL}, P<0.001$ and $P=0.045$, resp.) (Figure $1(\mathrm{f})$ ). Although we found significant alterations in several laboratory markers in complete or incomplete $\mathrm{KD}$, as mentioned above, there were no statistically significant differences between complete $\mathrm{KD}$ and incomplete KD patients' WBC counts, platelet counts, ESR, CRP, or serum albumin levels.

3.3. NT-proBNP Levels in Patients with KD and Simple Febrile Illness. In addition to the common serum chemical studies, we evaluated alterations in NT-proBNP levels. In all ages combined, the mean NT-proBNP levels in the complete KD group $(1785 \pm 2858 \mathrm{pg} / \mathrm{mL})$ and incomplete $\mathrm{KD}$ group $(1153 \pm 1725 \mathrm{pg} / \mathrm{mL})$ were significantly higher than that in the control group $(311 \pm 400 \mathrm{pg} / \mathrm{mL}, P<0.001$ for both) (Table 1, Figure 2(a)). The mean NT-proBNP level in the complete $\mathrm{KD}$ group was higher than that in the incomplete $\mathrm{KD}$ group $(P=0.033)$. We further investigated whether the aberrant elevation of NT-proBNP was tightly linked with inflammatory biomarkers such as CRP, platelet count, and elevated liver enzymes. To adjust for confounding factors, we performed a logistic regression between the clinical markers as well as NT-proBNP in the KD groups (Table 3). There were no statistically significant interactions between NT-proBNP, CRP, platelet count, and serum albumin. NT-proBNP was an independent predictor of $\mathrm{KD}$ despite the adjustment for CRP, platelet, and albumin levels $(P<0.001)$. Therefore, a high NT-proBNP level instead of a high CRP level increases the likelihood of a diagnosis of $\mathrm{KD}$.

3.4. NT-proBNP Level by Age. It has been reported that serum NT-proBNP is negatively correlated with a child's age [8]. Thus, we tested whether the sample distribution was closely linked with age using a bivariate correlation test. The serum NT-proBNP level tended to decrease as age increased (Pearson's correlation, $P=0.015$; Spearman's rho, $P<0.001$ ) (Figure 2(b)). Hence, we divided the patients into four groups to account for the effect of age and performed specific 
TABLE 2: Laboratory findings at diagnosis in patients with complete and incomplete Kawasaki disease (KD) and simple febrile illness (control) by age.

\begin{tabular}{|c|c|c|c|c|c|c|}
\hline & \multicolumn{2}{|c|}{ Complete KD } & \multicolumn{2}{|c|}{ Incomplete $\mathrm{KD}$} & \multicolumn{2}{|r|}{ Control } \\
\hline & $n$ & Value & $n$ & Value & $n$ & Value \\
\hline \multicolumn{7}{|l|}{$<6$ months } \\
\hline$n(\%)$ & 21 & $21(9.8)$ & 25 & $25(19.4)$ & 8 & $8(12.9)$ \\
\hline Age (years) & 21 & $0.4 \pm 0.1$ & 25 & $0.3 \pm 0.1$ & 8 & $0.3 \pm 0.2$ \\
\hline White blood cell (count $/ \mathrm{mm}^{3}$ ) & 21 & $13,776 \pm 4361$ & 25 & $16,460 \pm 6412$ & 8 & $13,338 \pm 6342$ \\
\hline Erythrocyte sedimentation rate $(\mathrm{mm} / \mathrm{h})$ & 13 & $42.7 \pm 21.8$ & 7 & $65.6 \pm 17.6$ & 3 & $37.3 \pm 20.5$ \\
\hline C-reactive protein (mg/dL) & 21 & $8.1 \pm 5.9$ & 25 & $8.7 \pm 4.7$ & 8 & $3.3 \pm 2.7^{\ddagger}$ \\
\hline N-terminal prohormone of brain natriuretic peptide $(\mathrm{pg} / \mathrm{mL})$ & 21 & $3404 \pm 3844$ & 25 & $1846 \pm 1811$ & 8 & $669 \pm 660^{*}$ \\
\hline \multicolumn{7}{|l|}{$6-12$ months } \\
\hline$n(\%)$ & 20 & $20(9.3)$ & 22 & $22(17.1)$ & 11 & $11(17.7)$ \\
\hline Age (years) & 20 & $0.8 \pm 0.2$ & 22 & $0.7 \pm 0.1$ & 11 & $0.8 \pm 0.2$ \\
\hline White blood cell (count $/ \mathrm{mm}^{3}$ ) & 20 & $16,360 \pm 3960$ & 22 & $13,250 \pm 6981$ & 11 & $10,982 \pm 3631^{*}$ \\
\hline Erythrocyte sedimentation rate $(\mathrm{mm} / \mathrm{h})$ & 10 & $75.3 \pm 28.6$ & 7 & $54.0 \pm 34.9$ & 2 & $2.0 \pm 0.0^{*}$ \\
\hline C-reactive protein (mg/dL) & 20 & $7.1 \pm 4.6$ & 22 & $4.7 \pm 5.2$ & 11 & $3.7 \pm 4.6$ \\
\hline N-terminal prohormone of brain natriuretic peptide $(\mathrm{pg} / \mathrm{mL})$ & 20 & $2241 \pm 3322$ & 22 & $882 \pm 1381$ & 11 & $301 \pm 310^{\dagger}$ \\
\hline \multicolumn{7}{|l|}{$12-24$ months } \\
\hline$n(\%)$ & 39 & $39(18.2)$ & 21 & $21(16.3)$ & 12 & $12(19.4)$ \\
\hline Age (years) & 39 & $1.6 \pm 0.3$ & 21 & $1.5 \pm 0.3$ & 12 & $1.6 \pm 0.3$ \\
\hline White blood cell (count $/ \mathrm{mm}^{3}$ ) & 39 & $14,341 \pm 4611$ & 21 & $12,886 \pm 4688$ & 12 & $12,967 \pm 3444$ \\
\hline Erythrocyte sedimentation rate $(\mathrm{mm} / \mathrm{h})$ & 17 & $64.5 \pm 23.8$ & 7 & $59.6 \pm 30.5$ & 4 & $39.8 \pm 38.2$ \\
\hline C-reactive protein (mg/dL) & 39 & $6.4 \pm 4.2$ & 21 & $4.6 \pm 4.4$ & 12 & $4.5 \pm 4.2$ \\
\hline $\mathrm{N}$-terminal prohormone of brain natriuretic peptide $(\mathrm{pg} / \mathrm{mL})$ & 39 & $1372 \pm 1387$ & 21 & $1297 \pm 1951$ & 12 & $398 \pm 560^{\dagger, 9}$ \\
\hline \multicolumn{7}{|l|}{$>24$ months } \\
\hline$n(\%)$ & 134 & $134(62.6)$ & 61 & $61(47.3)$ & 31 & $31(50.0)$ \\
\hline Age (years) & 134 & $3.8 \pm 1.5$ & 61 & $4.0 \pm 1.6$ & 31 & $4.9 \pm 2.5$ \\
\hline White blood cell (count/mm³) & 134 & $14,479 \pm 5096$ & 61 & $13,551 \pm 4657$ & 31 & $12,177 \pm 5166$ \\
\hline Erythrocyte sedimentation rate $(\mathrm{mm} / \mathrm{h})$ & 49 & $67.8 \pm 26.0$ & 29 & $76.2 \pm 34.9$ & 12 & $37.8 \pm 34.2^{*, \ddagger}$ \\
\hline C-reactive protein $(\mathrm{mg} / \mathrm{dL})$ & 134 & $8.8 \pm 5.5$ & 61 & $7.9 \pm 6.0$ & 31 & $5.8 \pm 5.8^{\dagger}$ \\
\hline N-terminal prohormone of brain natriuretic peptide $(\mathrm{pg} / \mathrm{mL})$ & 134 & $1583 \pm 2863$ & 61 & $916 \pm 1675^{\dagger}$ & 31 & $188 \pm 154^{\dagger, g}$ \\
\hline
\end{tabular}

KD: Kawasaki disease. Data are shown as mean \pm SD. ${ }^{*} P<0.05 .{ }^{\dagger} P<0.01$ versus complete KD. ${ }^{9} P<0.05 .{ }^{\ddagger} P<0.01$ versus incomplete KD.

comparisons of the groups (<6 months, 6-12 months, 1224 months, and $>24$ months). In the subgroups, the mean NT-proBNP level of patients with complete KD was significantly greater than that of age-matched controls. The specific distribution (Figure 3) and descriptive statistics (Table 2) are provided.

In patients $<6$ months of age, the mean NT-proBNP level in the complete KD group $(3404 \pm 3844 \mathrm{pg} / \mathrm{mL})$ was significantly higher than that in the control group $(669 \pm$ $660 \mathrm{pg} / \mathrm{mL}, P=0.011$ ) (Table 2, Figure 3(a)). Next, in patients 6-12 months of age, the mean NT-proBNP level in the complete KD group $(2241 \pm 3322 \mathrm{pg} / \mathrm{mL})$ was significantly higher than that in the control group $(301 \pm 310 \mathrm{pg} / \mathrm{mL}$, $P=0.002$; Table 2, Figure $3(\mathrm{~b}))$. In patients $12-24$ months of age, the mean NT-proBNP levels in the complete KD group $(1372 \pm 1387 \mathrm{pg} / \mathrm{mL})$ and incomplete $\mathrm{KD}$ group $(1297 \pm 1951 \mathrm{pg} / \mathrm{mL})$ were significantly higher than that in the control group $(398 \pm 560 \mathrm{pg} / \mathrm{mL}, P=0.004$ and $P=$ 0.024 , resp.) (Table 2, Figure 3(c)). Finally, in the group $>24$ months of age, the mean NT-proBNP levels in the complete $\mathrm{KD}$ group $(1583 \pm 2863 \mathrm{pg} / \mathrm{mL})$ and the incomplete $\mathrm{KD}$ group $(916 \pm 1675 \mathrm{pg} / \mathrm{mL})$ were significantly higher than that in the control group $(188 \pm 154 \mathrm{pg} / \mathrm{mL}, P<0.001$ and $P=$ 0.020 , resp.) (Table 2, Figure $3(\mathrm{~d}))$. The mean NT-proBNP level in the complete $\mathrm{KD}$ group was also higher than that in the incomplete $\mathrm{KD}$ group $(P=0.001)$.

Based on Pearson's correlation coefficient, we next postulated that the NT-proBNP level in younger patients with $\mathrm{KD}$ was also higher than that in older $\mathrm{KD}$ patients. The mean NT-proBNP level in the complete KD group $<6$ months of age was higher than that in the group $>24$ months of age $(P=0.002$; Figure $4(\mathrm{a}))$. The mean NT-proBNP level in the incomplete $\mathrm{KD}$ group $<6$ months of age was higher than those in the groups 6-12 months of age and $>24$ months of age $(P=0.018$ and $P<0.001$, resp.; Figure $4(b))$. The mean NT-proBNP level in the control group $<6$ months was higher than that in the group $>24$ months of age $(P=0.041$; Figure $4(c))$. 


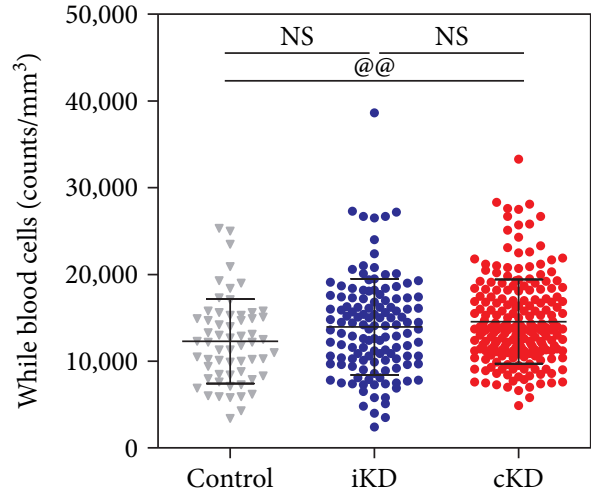

(a)

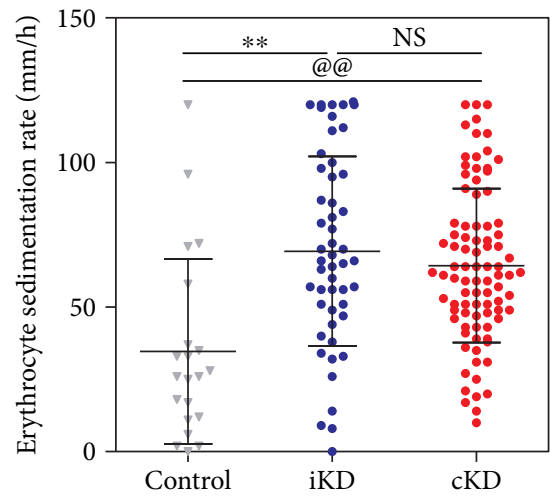

(d)

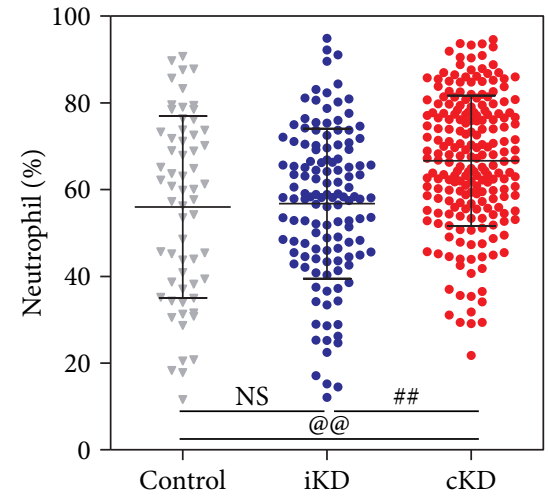

(b)

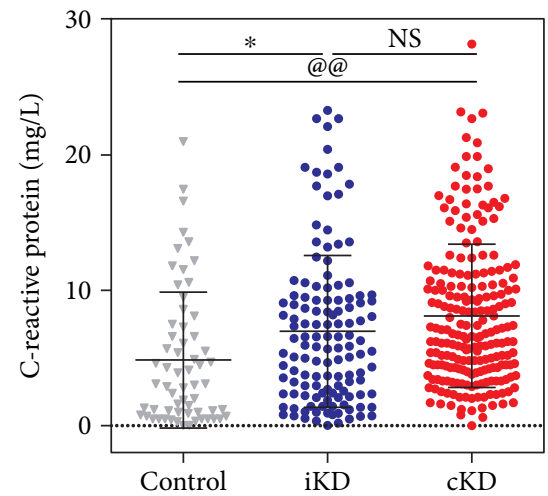

(e)

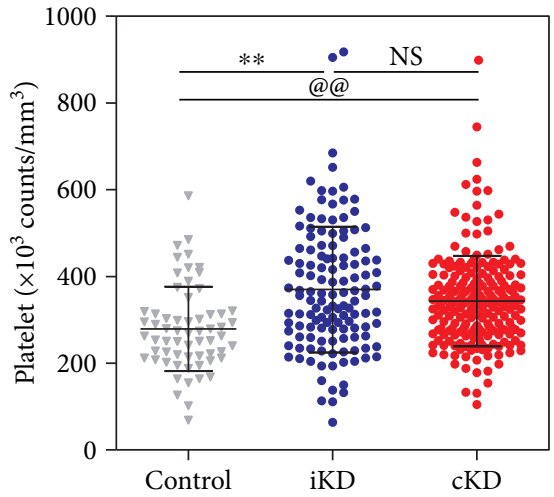

(c)

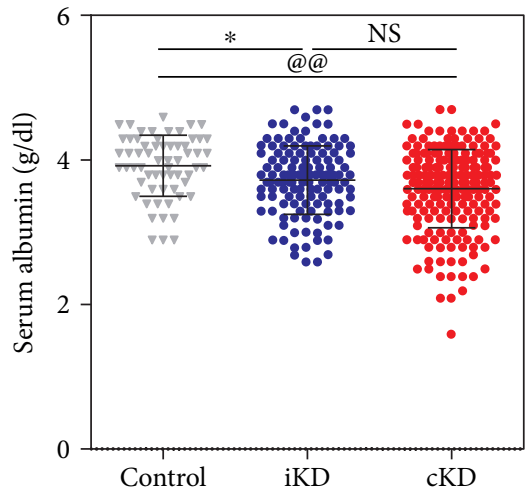

(f)

Figure 1: Dot plots of white blood cells (a), neutrophil percentage (b), platelet count (c), erythrocyte sedimentation rate (d), C-reactive protein level (e), and serum albumin level (f) in patients with complete Kawasaki disease (cKD), incomplete KD (iKD), and febrile illness (control). ${ }^{*} P<0.05 .{ }^{* *},{ }^{@}$, and ${ }^{\# \#} P<0.01$. NS: not significant.

Total age

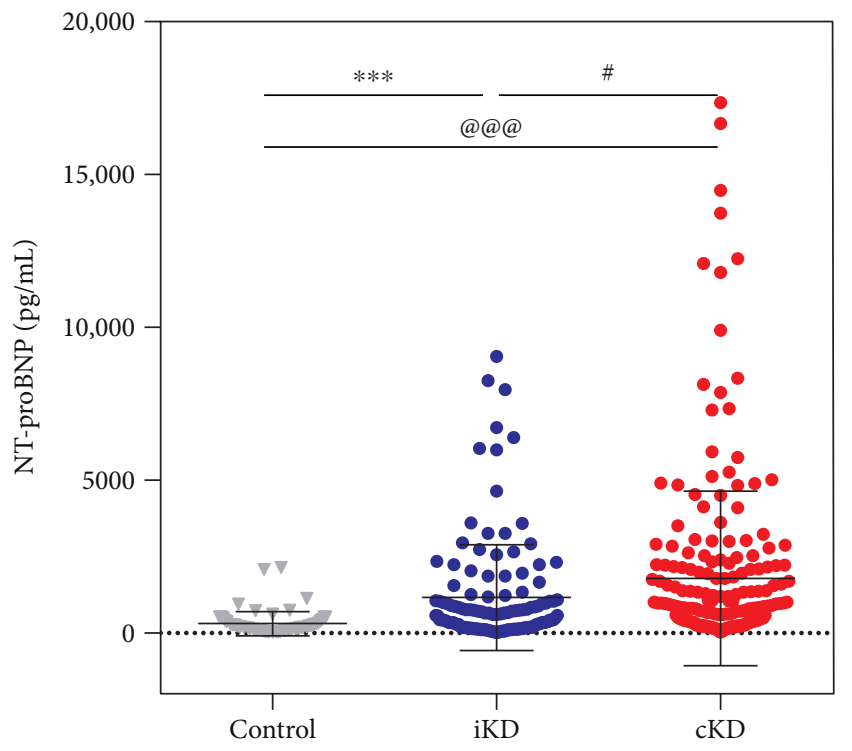

(a)

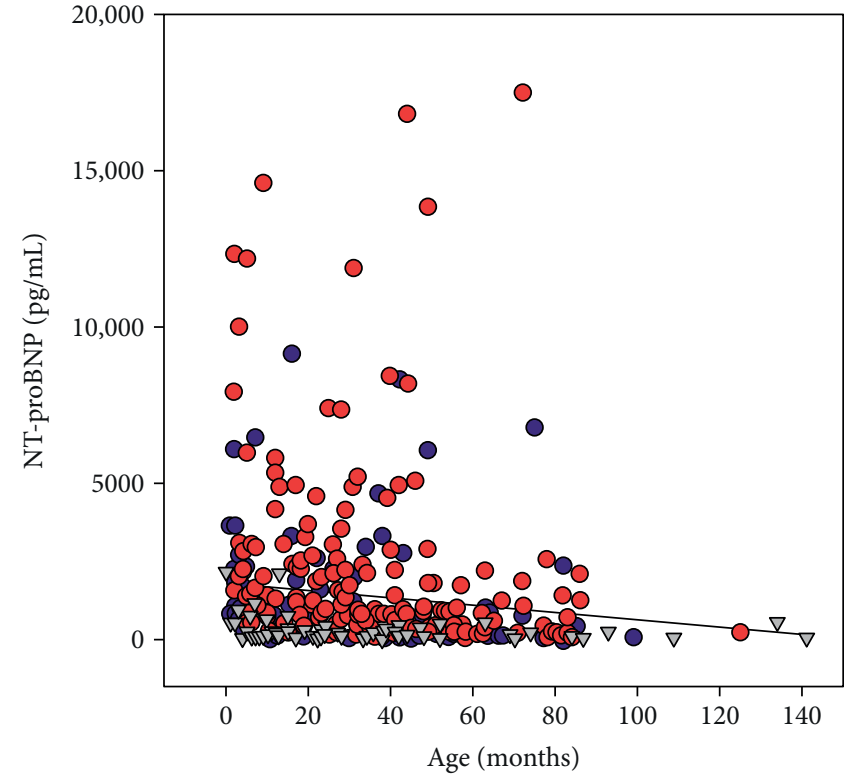

(b)

FIGURE 2: Dot plots of N-terminal prohormone of brain natriuretic peptide (NT-proBNP) in patients with complete Kawasaki disease (cKD), incomplete KD (iKD), and febrile illness (control) (a). NT-proBNP values by age (b). Black solid line depicts linear regression. ${ }^{\#} P<0.05$. *** and @@@ $P<0.001$. 
TABLe 3: Assessment of the associations between clinical markers and Kawasaki disease using multinomial logistic regression.

\begin{tabular}{lcc}
\hline Clinical marker & $\chi^{2}$ & $P$ value \\
\hline N-terminal prohormone & 30.52 & $<0.001$ \\
of brain natriuretic peptide & 1.64 & 0.441 \\
C-reactive protein & 30.03 & $<0.001$ \\
Platelet & 4.79 & 0.091 \\
Albumin & &
\end{tabular}

3.5. Cutoff Value of NT-proBNP for Diagnosing KD. Because the NT-proBNP level decreases with age, there is a need for different cutoff values to diagnose KD by patient age; thus, we used ROC curves to determine these values (Table 4). We found the cutoff value of NT-proBNP in patients with complete $\mathrm{KD}$, patients with incomplete $\mathrm{KD}$, and all $\mathrm{KD}$ patients compared with the simple febrile illness control group.

The cutoff value in the total KD patient group of all ages was $289 \mathrm{pg} / \mathrm{mL}$, with $71.7 \%$ sensitivity and $71.9 \%$ specificity (area under the curve $(\mathrm{AUC})=0.774)$. The cutoff value in the group aged $<6$ months was $762 \mathrm{pg} / \mathrm{mL}$, with $73.9 \%$ sensitivity and $75.0 \%$ specificity $(\mathrm{AUC}=0.813)$. Similarly, the cutoff values in the other age groups (6-12 months, $12-24$ months, and $>24$ months) were $310 \mathrm{pg} / \mathrm{mL}$ (sensitivity, $78.6 \%$; specificity, $81.8 \%$; $\mathrm{AUC}=0.779), 326 \mathrm{pg} / \mathrm{mL}$ (sensitivity, 76.7\%; specificity, 75.0\%; AUC $=0.796$ ), and $208 \mathrm{pg} / \mathrm{mL}$ (sensitivity, 70.8\%; specificity, 69.7\%; AUC= 0.782 ), respectively. Cutoff values were also obtained in the complete KD patient group. The cutoff value in the complete $\mathrm{KD}$ group of all ages was $326 \mathrm{pg} / \mathrm{mL}$, with $73.8 \%$ sensitivity and $74.2 \%$ specificity (AUC $=0.795$ ), while that in the group aged $<6$ months was $821 \mathrm{pg} / \mathrm{mL}$, with $71.4 \%$ sensitivity and $75.0 \%$ specificity $(\mathrm{AUC}=0.810)$. The cutoff values in the other age groups (6-12 months, 12-24 months, and $>24$ months) were $315 \mathrm{pg} / \mathrm{mL}$ (sensitivity, $85.0 \%$; specificity, 81.8\%; AUC $=0.868$ ), $330 \mathrm{pg} / \mathrm{mL}$ (sensitivity, $74.4 \%$; specificity, $75.0 \%$; AUC = 0.797), and $255 \mathrm{pg} / \mathrm{mL}$ (sensitivity, $73.1 \%$; specificity, $77.4 \%$; AUC $=0.836$ ), respectively. Similar cutoff values were obtained in the incomplete $\mathrm{KD}$ patient group. The cutoff value in the incomplete $\mathrm{KD}$ group of all ages was $261 \mathrm{pg} / \mathrm{mL}$, with $68.8 \%$ sensitivity and $67.2 \%$ specificity $(\mathrm{AUC}=0.719)$, and the cutoff value in the group aged $<6$ months was $762 \mathrm{pg} / \mathrm{mL}$, with $76.0 \%$ sensitivity and $75.0 \%$ specificity $(A U C=0.815)$. The cutoff values in the other age groups (6-12 months, 12-24 months, and $>24$ months) were $310 \mathrm{pg} / \mathrm{mL}$ (sensitivity, $72.7 \%$; specificity, $81.8 \%$; AUC $=0.698$ ), $326 \mathrm{pg} / \mathrm{mL}$ (sensitivity, $81.0 \%$; specificity, $75.0 \%$; AUC $=0.794$ ), and $137 \mathrm{pg} / \mathrm{mL}$ (sensitivity, $70.5 \%$; specificity, $54.5 \%$; AUC $=0.665)$, respectively.

\section{Discussion}

Because a definitive diagnostic test for $\mathrm{KD}$ does not exist, diagnosis depends on a combination of clinical and laboratory findings [10]. Therefore, diagnosing $\mathrm{KD}$ is challenging in patients who show nonspecific symptoms or do not fulfill all of the criteria [11]. For this reason, many adjuvant diagnostic markers have been proposed, such as cytokines like helper T-cell (Th) 1, Th 2, interleukin- (IL-) 6, IL-20, tumor necrosis factor- (TNF-) alpha, and NT-proBNP [9].

Pre-proBNP, an analog of NT-proBNP, consists of 134 amino acids and is transformed into proBNP (108 amino acids), which is released from cardiac myocytes into the bloodstream. It is then broken down into bioactive BNP (32 amino acids) and NT-proBNP (76 amino acids). Usually, NT-proBNP plays no function in the body, but it can be used to indirectly measure the level of bioactive BNP [6]. Because of its stability and longer half-life, NT-proBNP is easier to use than bioactive BNP $[7,12]$. Bioactive BNP and NT-proBNP have already been used to predict patient prognosis in adults with left ventricular dysfunction, ischemic heart disease, and hypertrophic cardiomyopathy [13]. Also, because it is positively related to the severity of congestive heart failure and left ventricular dysfunction, NT-proBNP is a useful biomarker in patients with cardiac disease [13].

Here, we found that the mean NT-proBNP in complete and incomplete KD patients was significantly higher than that in simple febrile illness patients in all age-stratified subgroups. Also, the mean NT-proBNP in complete KD patients was higher than that in incomplete patients. Our data revealed that the cutoff values for diagnosing KD and incomplete $\mathrm{KD}$ were $289 \mathrm{pg} / \mathrm{mL}(71.7 \%$ sensitivity and $71.0 \%$ specificity) and $261 \mathrm{pg} / \mathrm{mL}$ ( $72.1 \%$ sensitivity and $74.2 \%$ specificity), respectively. Many previous studies suggested cutoff values for distinguishing $\mathrm{KD}$ or incomplete $\mathrm{KD}$ from simple febrile illness $[6,7,14]$. Cho et al. recently reported that NT-proBNP might be a more useful marker than highsensitivity CRP (hs-CRP) for diagnosing KD [14]. Those authors found that NT-proBNP levels were significantly higher in $59 \mathrm{KD}$ patients $(749.66 \pm 997.11 \mathrm{pg} / \mathrm{mL})$ than in 45 febrile illness patients used as age-matched controls $(174.41 \pm 144.30 \mathrm{pg} / \mathrm{mL}, P<0.001)$ and that the differential diagnostic cutoff value of NT-proBNP was $235.2 \mathrm{pg} / \mathrm{mL}$, with a sensitivity of $66.10 \%$ and a specificity of $77.08 \%$. We also found limitations of using CRP to diagnose KD. Although an elevated CRP level is widely accepted as a common laboratory finding in $\mathrm{KD}$ patients, we found that an increase in CRP is not an independent marker but, rather, a confounder on logistic regression analysis. On the contrary, NT-proBNP itself was a powerful diagnostic marker of $\mathrm{KD}$ and useful in distinguishing it from simple febrile illness.

Lee et al. also investigated the cutoff level of NT proBNP that allowed them to distinguish 33 incomplete KD patients from 19 simple febrile illness patients. They proposed that an NT-proBNP cutoff value of $158 \mathrm{pg} / \mathrm{mL}$ provided a sensitivity of $81 \%$ and a specificity of $74 \%$ for the diagnosis of incomplete KD [6]. Another study reported that the cutoff value of NT-proBNP for diagnosing KD was $260 \mathrm{pg} / \mathrm{mL}$, with $93 \%$ sensitivity and $88 \%$ specificity, through a comparison of $58 \mathrm{KD}$ patients and 34 febrile illness patients [7].

However, previous studies did not consider the natural changes that occur in NT-proBNP levels with age. Here, we considered patient age a confounder and evaluated the different cutoff values for each disease group and the age-stratified subgroup. In each patient group, the mean NT-proBNP level decreased as age increased. In all $\mathrm{KD}$ patients, the 


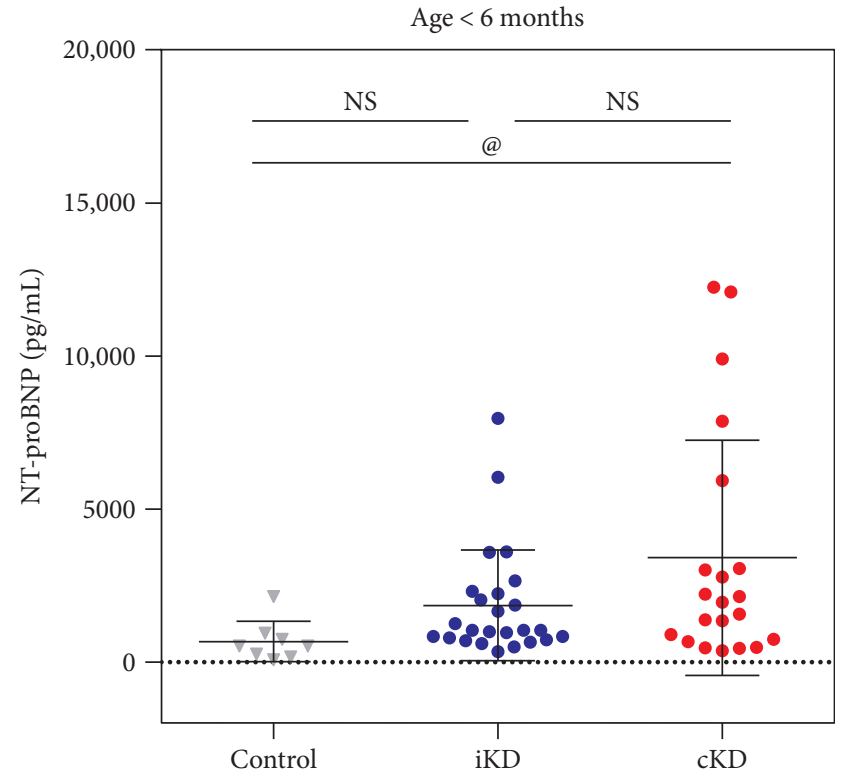

(a)

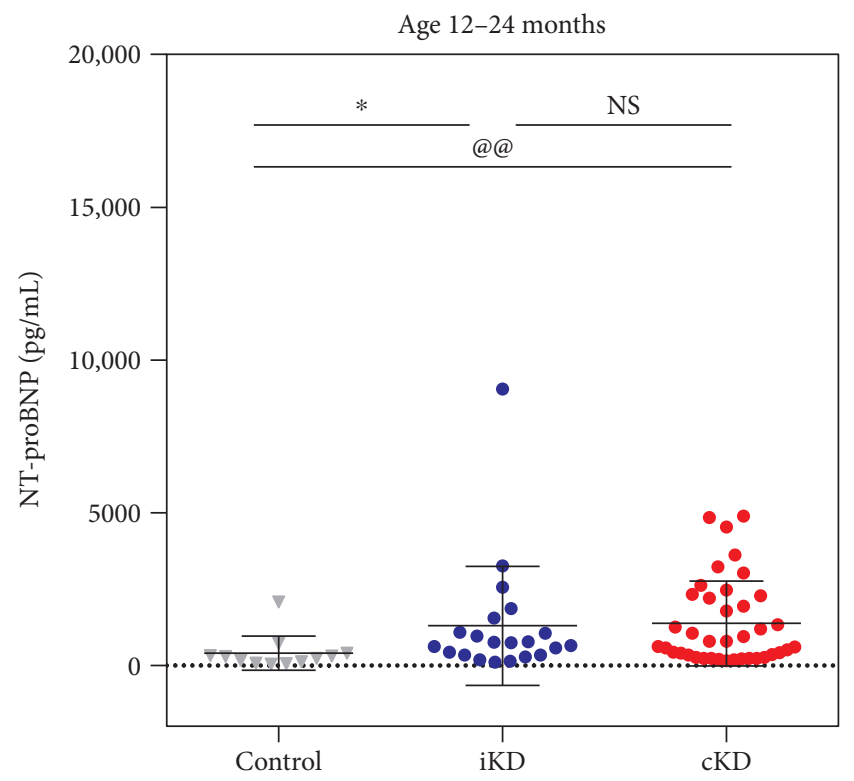

(c)

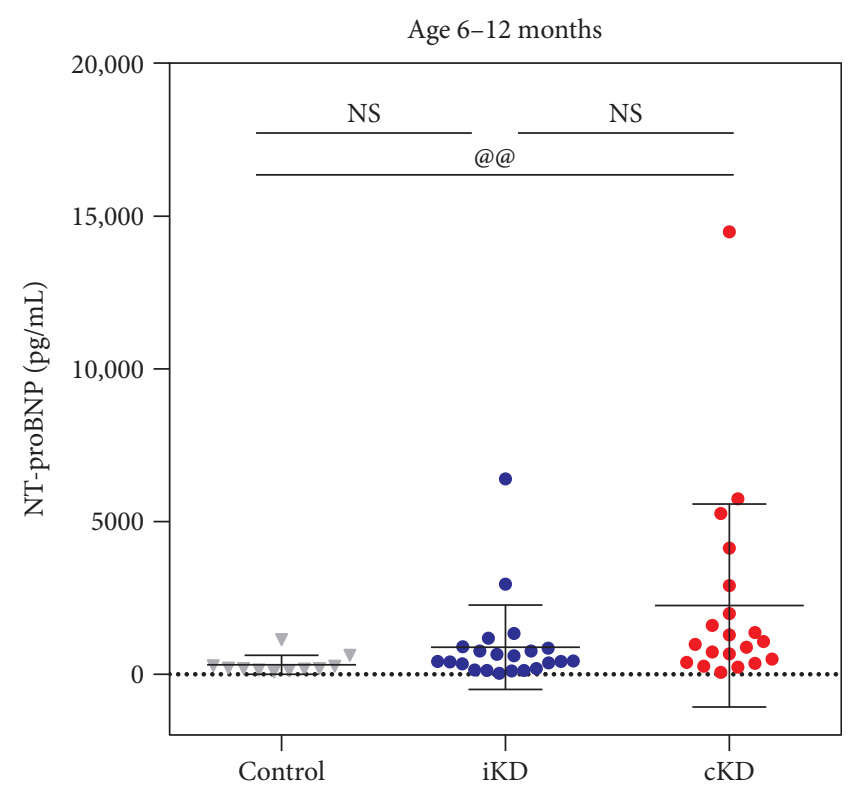

(b)

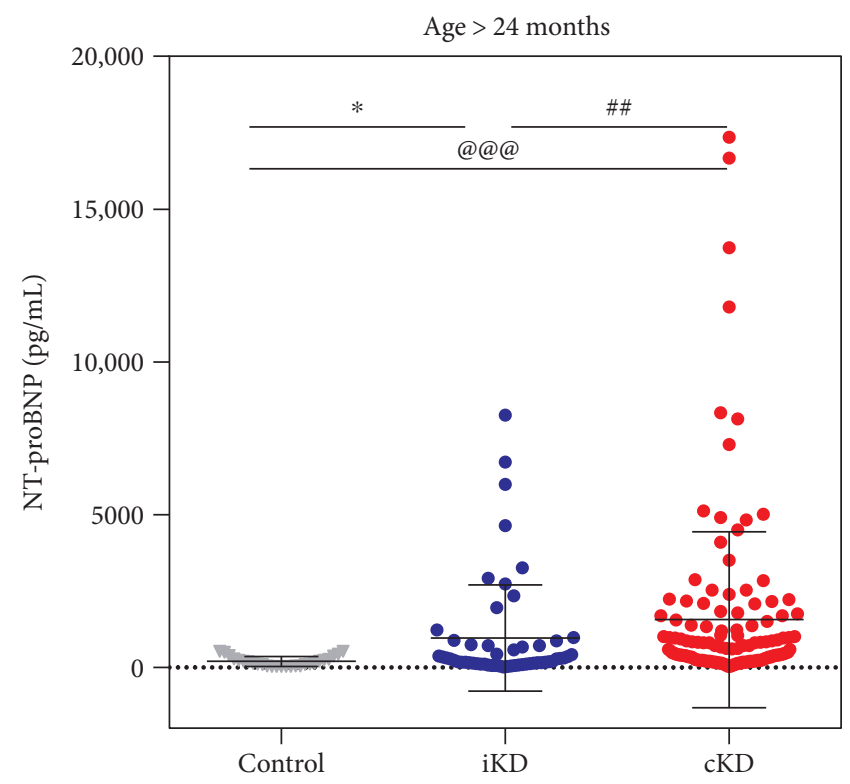

(d)

FIgURe 3: Dot plot of N-terminal prohormone of brain natriuretic peptide (NT-proBNP) in patients with complete Kawasaki disease (KD), incomplete KD, and febrile illness (control) according to age. ${ }^{*}$ and ${ }^{\circledR} P<0.05$. ${ }^{@ @}$ and ${ }^{\# \#} P<0.01$. ${ }^{@ @} P<0.001$. NS: not significant.

cutoff value of NT-proBNP was $289 \mathrm{pg} / \mathrm{mL}$ for all age groups and $762 \mathrm{pg} / \mathrm{mL}$ for those aged $<6$ months. In the incomplete KD group, the cutoff value of NT-proBNP was $261 \mathrm{pg} / \mathrm{mL}$ in all ages and $762 \mathrm{pg} / \mathrm{mL}$ in those aged $<6$ months. In children $<6$ months of age, applying the same NT-proBNP cutoff value for diagnosing KD regardless of age resulted in a higher sensitivity but a significantly lower specificity. Therefore, the usefulness of NT-proBNP as a diagnostic marker for KD was decreased.

We also found that the age-stratified cutoff values for patients aged 6-12 months were not significantly different from those for patients aged 12-24 months, including in terms of sensitivity and specificity. Therefore, for diagnosing
KD based on NT-proBNP level, dividing the patients into three groups by age ( $<6$ months, 6-24 months, and $>24$ months) and applying different cutoff values are more useful.

There are a few limitations to our study. We used a retrospective design and included a relatively small number of simple febrile illness patients as the control group. The major enrollment criterion for the control group was the performance of an NT-proBNP test. However, because of its high cost and need for extra blood samples, we did not perform an NT-proBNP test on every child with febrile illness who visited our center. Furthermore, we found relatively low sensitivity and specificity of the cutoff values compared to those in previous studies, especially in the group of 


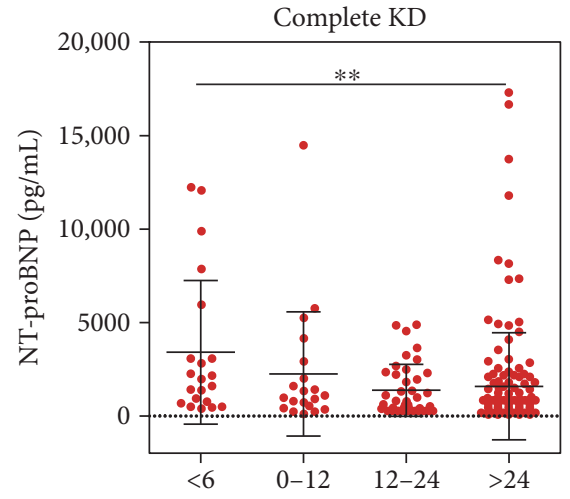

(a)

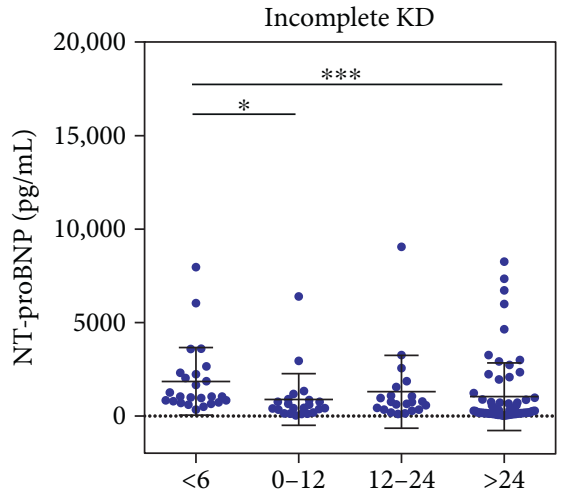

(b)

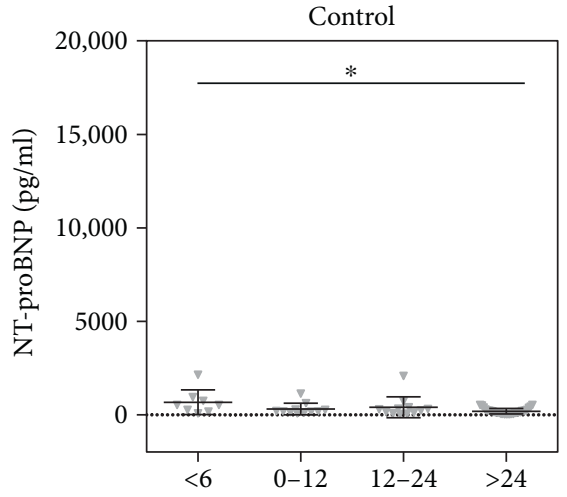

(c)

FIGURE 4: Dot plot of N-terminal prohormone of brain natriuretic peptide (NT-proBNP) in different age groups in patients with complete Kawasaki disease (KD) (a), incomplete KD (b), and febrile illness (control) (c). ${ }^{*} P<0.05 .{ }^{* *} P<0.01 .{ }^{* * *} P<0.001$. Otherwise, not significant.

TABLE 4: Diagnostic cutoff values of N-terminal prohormone of brain natriuretic peptide (NT-proBNP) in patients with complete, incomplete, and total (complete and incomplete) Kawasaki Disease (KD) compared to patients with simple febrile illness.

\begin{tabular}{lccc}
\hline & $\begin{array}{c}\text { Cutoff value of } \\
\text { NT-proBNP } \\
(\mathrm{pg} / \mathrm{mL})\end{array}$ & Sensitivity (\%) & Specificity (\%) \\
\hline Complete KD & & & \\
<6 months & 821 & 85.0 & 75.0 \\
6-12 months & 315 & 74.4 & 75.8 \\
12-24 months & 330 & 73.1 & 77.4 \\
$>$ 24 months & 255 & 73.8 & 74.2 \\
All ages & 326 & & \\
Incomplete KD & & 76.0 & 75.0 \\
$<6$ months & 762 & 72.7 & 81.8 \\
6-12 months & 310 & 81.0 & 75.0 \\
12-24 months & 326 & 70.5 & 54.5 \\
$>24$ months & 137 & 68.8 & 67.2 \\
All ages & 261 & & \\
Total KD & & 73.9 & 75.0 \\
$<6$ months & 762 & 78.6 & 81.8 \\
6-12 months & 310 & 76.7 & 75.0 \\
12-24 months & 326 & 70.8 & 69.7 \\
$>$ 24 months & 208 & 71.7 & 71.9 \\
All ages & 289 & & \\
\hline
\end{tabular}

patients $>24$ months with incomplete KD $[6,7]$. Further, large prospective investigations are required for selecting higher cutoffs of NT-proBNP as a diagnostic biomarker. Also, the NT-proBNP value has limited usefulness in patients with complete $\mathrm{KD}$, which has classical diagnostic criteria. However, NT-proBNP was an independent predictor of KD despite the adjustment for CRP, platelet, and albumin levels $(P<0.001)$. The onsets of each clinical symptom differed by the diagnostic criteria. Thus, NT-proBNP can be a useful adjuvant marker for early diagnosis. Finally, the cutoff values were most useful for distinguishing incomplete KD from simple febrile illness patients $<6$ months of age. However, the incidence of the disease in young infants is very low; in our center, only 25 patients were diagnosed over a 9 -year period. Therefore, the clinical advantage of cutoff values obtained in this manner may be limited by the low disease incidence.

Here, we proposed cutoff values of NT-proBNP in different age groups that were useful for diagnosing complete and incomplete $\mathrm{KD}$ and distinguishing those conditions from simple febrile illness. For differentiating any form of $\mathrm{KD}$ from febrile illness, the cutoff values are $762 \mathrm{pg} / \mathrm{mL}$ (<6 months), $310 \mathrm{pg} / \mathrm{mL}$ (6-12 months), $326 \mathrm{pg} / \mathrm{mL}$ (12-24 months), and $208 \mathrm{pg} / \mathrm{mL}$ (>24 months). For diagnosing incomplete $\mathrm{KD}$, the cutoff values are $762 \mathrm{pg} / \mathrm{mL}$ ( $<6$ months), $310 \mathrm{pg} / \mathrm{mL}$ (6-12 months), $326 \mathrm{pg} / \mathrm{mL}$ (12-24 months), and $137 \mathrm{pg} / \mathrm{mL}$ (>24 months).

In conclusion, this is the first report of age-stratified measurements of NT-proBNP in patients with complete or incomplete $\mathrm{KD}$ and controls with simple febrile illness. Age-stratified NT-proBNP is a useful biomarker for the differential diagnosis of complete $\mathrm{KD}$ and incomplete $\mathrm{KD}$ from simple febrile illness.

\section{Conflicts of Interest}

The authors have no conflicts of interest to disclose.

\section{Authors' Contributions}

Sang Hoon Lee, Eun Song Song, and Somy Yoon contributed equally to the study.

\section{Acknowledgments}

The authors thank Professor Hee-Young Shin for his assistance with the statistical analysis. This study was supported by Grant NRF-2015R1D1A1A01059017 from the Basic Science Research Program through the National Research Foundation of Korea (NRF) funded by the Ministry of Education, Republic of Korea. 


\section{References}

[1] D. Yanagisawa, M. Ayusawa, M. Kato et al., "Factors affecting $\mathrm{N}$-terminal pro-brain natriuretic peptide elevation in the acute phase of Kawasaki disease," Pediatrics International, vol. 58, no. 11, pp. 1105-1111, 2016.

[2] K. Takahashi, T. Oharaseki, Y. Yokouchi, H. Yamada, K. Shibuya, and S. Naoe, "A half-century of autopsy resultsincidence of pediatric vasculitis syndromes, especially Kawasaki disease," Circulation Journal, vol. 76, no. 4, pp. 964970, 2012.

[3] K. H. Lin, S. S. Chang, C. W. Yu et al., "Usefulness of natriuretic peptide for the diagnosis of Kawasaki disease: a systematic review and meta-analysis," British Medical Journal Open, vol. 5, no. 4, article e006703, 2015.

[4] M. Toyono, D. Takagi, J. Oyamada, S. Shimada, M. AokiOkazaki, and T. Takahashi, "Coronary artery aneurysm after Kawasaki disease in a single coronary artery," Circulation Journal, vol. 77, no. 9, pp. 2409-2411, 2013.

[5] B. W. McCrindle, A. H. Rowley, J. W. Newburger et al., "Diagnosis, treatment, and long-term management of Kawasaki disease: a scientific statement for health professionals from the American Heart Association," Circulation, vol. 135, no. 17, pp. e927-e999, 2017.

[6] D. W. Lee, Y. H. Kim, M. C. Hyun, T. C. Kwon, and S. B. Lee, "NT-proBNP as a useful tool in diagnosing incomplete Kawasaki disease," Korean Journal of Pediatrics, vol. 53, no. 4, pp. 519-524, 2010.

[7] H. J. Lee, H. J. Kim, H. S. Kim, and S. J. Sohn, "NT-pro BNP: a new diagnostic screening tool for Kawasaki disease," Korean Journal of Pediatrics, vol. 49, no. 5, pp. 539-544, 2006.

[8] A. Nir, A. Lindinger, M. Rauh et al., "NT-pro-B-type natriuretic peptide in infants and children: reference values based on combined data from four studies," Pediatric Cardiology, vol. 30, no. 1, pp. 3-8, 2009.

[9] A. Rawat and S. Singh, "Biomarkers for diagnosis of Kawasaki disease," Indian Pediatrics, vol. 52, no. 6, pp. 473-474, 2015.

[10] D. Eleftheriou, M. Levin, D. Shingadia, R. Tulloh, N. J. Klein, and P. A. Brogan, "Management of Kawasaki disease," Archives of Disease in Childhood, vol. 99, no. 1, pp. 74-83, 2014.

[11] K. K. Seaton and A. Kharbanda, "Evidence-based management of Kawasaki disease in the emergency department," Pediatric Emergency Medicine Practice, vol. 12, no. 1, pp. 1-20, 2015.

[12] M. Weber and C. Hamm, "Role of B-type natriuretic peptide (BNP) and NT-proBNP in clinical routine," Heart, vol. 92, no. 6, pp. 843-849, 2006.

[13] I. Nevo, M. Erlichman, N. Algur, and A. Nir, "N-terminal pro B-type natriuretic peptide levels in infants and children with acute non-cardiac diseases," Israel Medical Association Journal, vol. 13, no. 7, pp. 420-424, 2011.

[14] S. Y. Cho, Y. Kim, S. H. Cha, J. T. Suh, M. Y. Han, and H. J. Lee, "Adjuvant laboratory marker of Kawasaki disease; NTpro-BNP or hs-CRP?," Annals of Clinical and Laboratory Science, vol. 41, no. 4, pp. 360-363, 2011. 


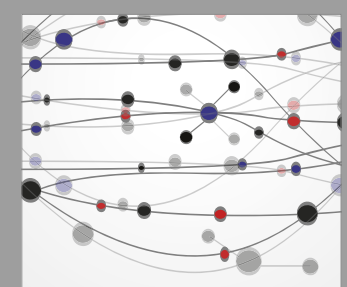

The Scientific World Journal
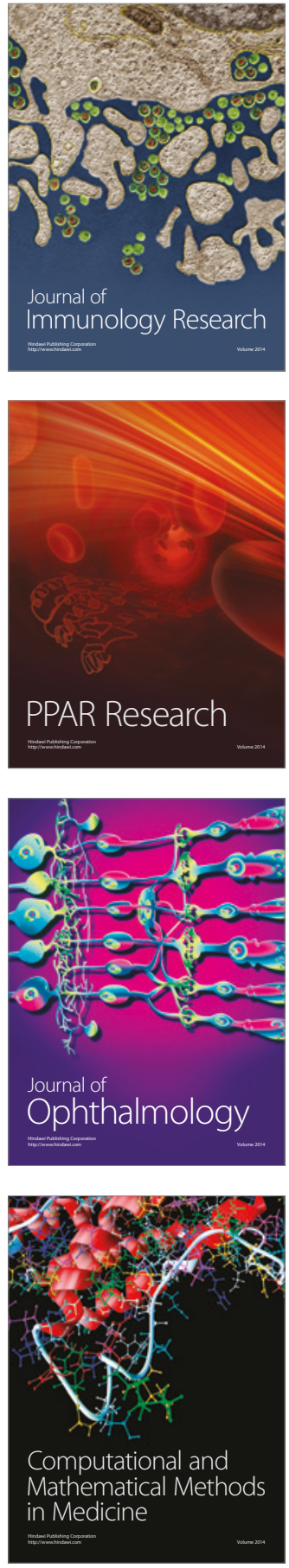

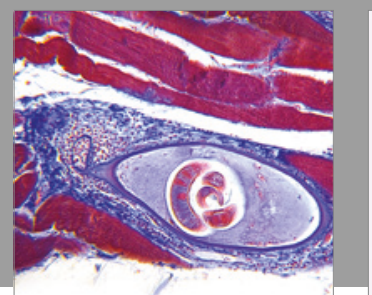

Gastroenterology Research and Practice
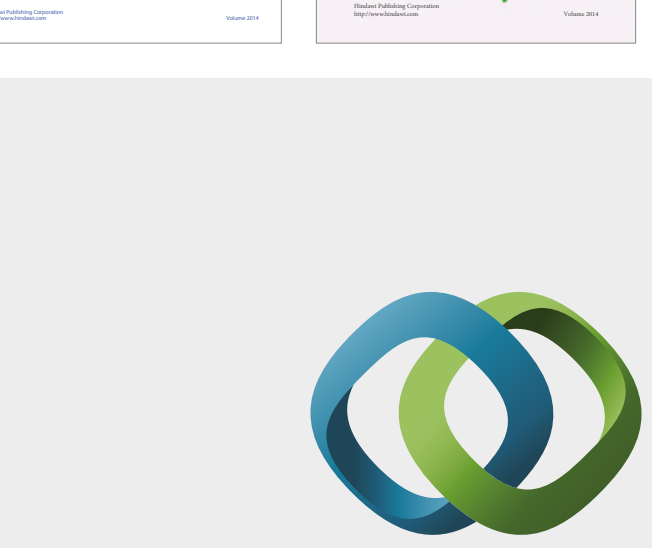

\section{Hindawi}

Submit your manuscripts at

https://www.hindawi.com
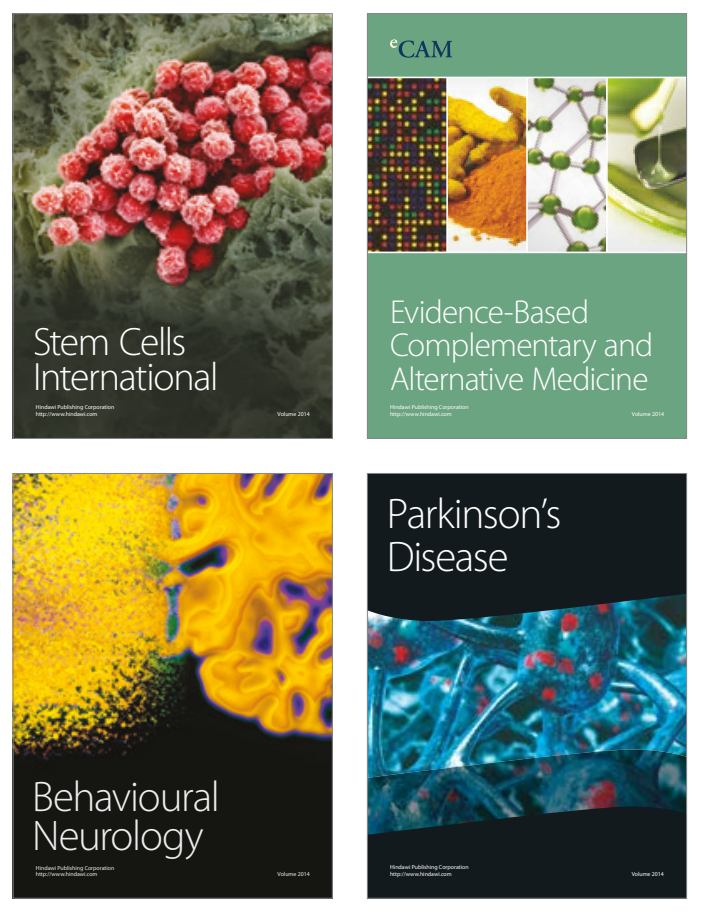
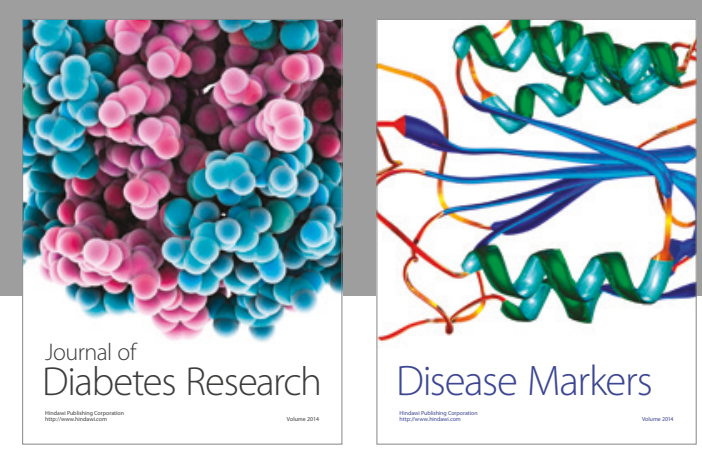

Disease Markers
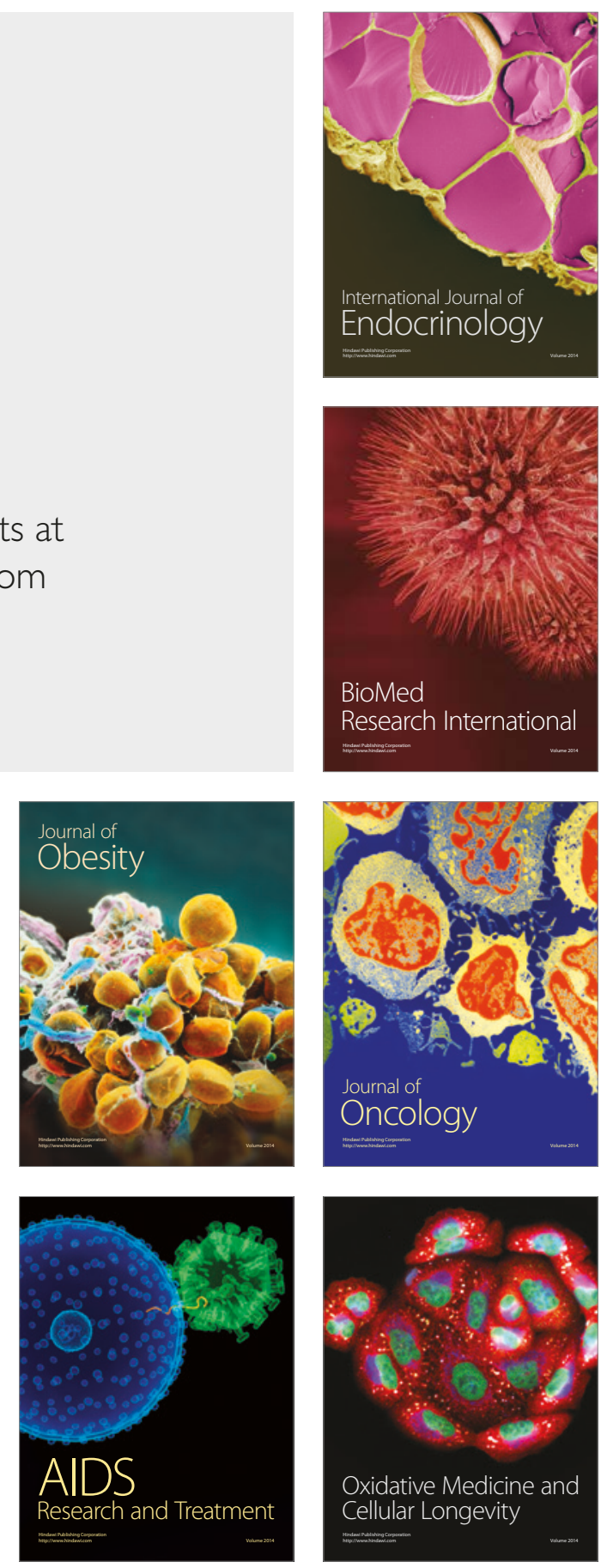